\title{
On local existence of the Vlasov-Fokker-Planck equation in a 2D anisotropic space
}

Jing Chun Chen ${ }^{1}$ and Cong $\mathrm{He}^{2^{*}}$

\section{*Correspondence:}

josephhecongcfa@gmail.com

${ }^{2}$ School of Mathematics and

Statistics, Wuhan University, Wuhan,

430072, P.R. China

Full list of author information is

available at the end of the article

\begin{abstract}
We are concerned with local existence of the Vlasov-Fokker-Planck equation in a $2 D$ anisotropic space $L_{x}^{p} L_{v}^{1}$ in a bounded domain with respect to the space variable. The energy method is used to construct the result and the Hardy inequality is used to estimate the electric field.
\end{abstract}

Keywords: Vlasov-Fokker-Planck equation; anisotropic space; Hardy inequality; energy method

\section{Introduction}

Through this paper, as in the title, when anisotropic space is mentioned, we mean that the space variable $x$ and the velocity variable $v$ have different regularities, such as integrability and differentiability. Firstly, we would like to give a review which has close relationship with our paper. As to a classical solution, it is worthy to mention the paper of Yang and $\mathrm{Yu}$ [1]. They constructed the global-in-time classical solutions to the Vlasov-MaxwellFokker-Planck system near Maxwellian using an approach by combining the compensating function and energy method. For the system of (1.1) in the whole space, there are many results about weak solutions. We refer to them as follows. Triolo [2] obtained the global (local) existence in 1 and 2-dimension (3-dimension) for the Cauchy problem of the Vlasov-Poisson-Fokker-Planck equation under the initial value $f_{0} \in L^{1} \cap L^{\infty} \cap C^{0}\left(\mathbb{R}^{n} \times \mathbb{R}^{n}\right)$, $n=1,2,3$. Neunzert and some other authors [3] considered the modified Vlasov-FokkerPlanck equation problem, under the condition that the initial value $f_{0} \in L^{\infty}\left(\mathbb{R}^{2} \times \mathbb{R}^{2}\right)$ has compact support in $v$, they got a solution which is a probability measure. Zheng and Majda [4] gave the existence of a global weak solution of the Vlasov-Fokker-Planck system under the measure-valued initial data. Degond [5] obtained the existence of solution which has the same regularity as the initial value

$$
f_{0} \in W^{1,1}\left(\mathbb{R}^{2 n}\right), \quad\left(1+|v|^{2}\right)\left(\left|f_{0}\right|+\left|D f_{0}\right|\right) \in L^{\infty} .
$$

The author required that $f_{0}$ has the same index of differentiability and integrability with respect to variables $x$ and $v$, i.e., they are isotropic. Comparing with [5], we do not need $f_{0}, D f_{0}$ to be bounded, continuous to offset, we cannot obtain the global existence even in a 2D space because of the difficulty to get the Gronwall inequality which can be solved in

○2013 Chen and He; licensee Springer. This is an Open Access article distributed under the terms of the Creative Commons Attribution License (http://creativecommons.org/licenses/by/2.0), which permits unrestricted use, distribution, and reproduction in any medium, provided the original work is properly cited. 
$t \in[0, \infty)$. Roughly speaking, the essential difficult point lies in that $\left\|\nabla_{x} E^{n} \cdot \nabla_{v} f^{n+1}\right\|_{L_{x}^{p} L_{v}^{1}}$ is in proportion to $\|f\|_{L_{x}^{p} L_{v}^{1}}^{2}$, which is super-linear.

In this paper, we consider the initial boundary problem with 0 boundary value (see (1.1)). The problem about general boundary value $[6,7]$ is so tough that we are going to consider it in the next paper. Moreover, we focus on the case in which the initial value $f_{0}$ has different regularities with respect to the $x$ and $v$ variables, i.e., they have different integral and differential indices. An anisotropic space is natural since the variables $x$ and $v$ need not to have the same regularity. For instance, Strain [8] considered the anisotropic space $\dot{B}_{2}^{-\varrho, \infty} L_{v}^{2}$ when he studied the optimal decay rate of the solution to the hard and soft potential Boltzmann equation.

Before narrating the main theorem, we would like to introduce some notations.

\section{Notation}

(i) $f(x, v, t), x \in \Omega \subset \mathbb{R}^{2}$ with $\partial \Omega \in C^{1}, v \in \mathbb{R}^{2}, t>0$, the distribution function of the particles, where $\Omega$ is a bound open set with a $C^{1}$ boundary (for Definition see p.626 of [9]).

(ii) $L_{x}^{p} L_{v}^{1}$ is a space in which the elements are given the norm $\left(\int_{\Omega}\|f\|_{L_{v}^{1}}^{p} d x\right)^{\frac{1}{p}}, 1 \leq p<\infty$, here $L_{v}^{1}$ is the Lebesgue space with the norm given by $\|f\|_{L_{v}^{1}}:=\int_{\mathbb{R}^{2}}|f| d v$.

(iii) $L^{2}\left([0, T] \times \Omega_{x}, H^{1}\left(\mathbb{R}_{v}^{2}\right)\right)$ is a space given the norm $\left(\int_{[0, T] \times \Omega_{x}}\|f\|_{H_{\nu}^{1}}^{2} d t d x\right)^{\frac{1}{2}}$, here $H_{v}^{1}$ is the Sobolev space with respect to $v$.

Now we are ready to state our main theorem.

In the theorem, we denote respectively by $E(x, t), \rho(x, t)$ the electric field and the electric charge. $\sigma>0$ is a diffusion coefficient which is very small in physical situations.

Theorem 1.1 Suppose $f_{0} \in L^{2}\left(\Omega \times \mathbb{R}_{v}^{2}\right)$ and $\left.\left\|f_{0}\right\|_{L_{\nu}^{1}}\right|_{\partial \Omega}=0$. Also, we assume that $f_{0}$ is nonnegative and

$$
\left\|f_{0}\right\|_{L_{x}^{p} L_{v}^{1}},\left\|\nabla_{v} f_{0}\right\|_{L_{x}^{p} L_{v}^{1}},\left\|\nabla_{x} f_{0}\right\|_{L_{x}^{p} L_{v}^{1}} \leq \text { const. }
$$

Here $p=\frac{4}{3}$. Then the Vlasov-Fokker-Planck system with bounded domain

$$
\left\{\begin{array}{l}
\frac{\partial f}{\partial t}+v \cdot \nabla_{x} f+\left.E\right|_{\Omega} \cdot \nabla_{v} f-\sigma \Delta_{v} f=0 ; \\
E(x, t)=\int_{\mathbb{R}^{2}} \frac{x-y}{|x-y|^{2}} \rho(y, t) d y \\
\rho(x, t)=\int_{\mathbb{R}^{2}} f(x, v, t) d v ; \\
f(x, v, 0)=f_{0}(x, v),\left.\quad\|f(x, v, t)\|_{L_{v}^{1}}\right|_{\partial \Omega}=0 \quad \text { for } t>0
\end{array}\right.
$$

admits a solution in the interval $[0, T)$, i.e., $f(x, v, t) \in L_{\mathrm{loc}}^{\infty}\left([0, T), L_{x}^{p} L_{v}^{1}\right)$. Here we mean the boundary value in the sense of trace (see p.259 of [9]). Moreover, we have that $\nabla_{x} f, \nabla_{v} f \in$ $L_{\text {loc }}^{\infty}\left([0, T), L_{x}^{p} L_{v}^{1}\right)$. The solution is unique.

The arrangement of this paper is the following. In Section 2, we cite or prove some lemmas which will be used in the proof of Theorem 1.1. In Section 3, we give the proof of the main theorem in several steps. 


\section{Some lemmas}

Consider the linear equation, i.e.,

$$
\frac{\partial f}{\partial t}+v \cdot \nabla_{x} f+\left.E\right|_{\Omega} \cdot \nabla_{v} f-\sigma \Delta_{v} f=U ; \quad f(x, v, 0)=f_{0}(x, v) .
$$

Next we introduce some lemmas, which are modified versions of the lemmas in Appendix A in [5] and will be used in the proof of Theorem 1.1 in Section 3. We only point out the differences, the details can be found in the reference.

Lemma 2.1 Assume

$$
\begin{aligned}
& f_{0} \in L^{2}\left(\Omega \times \mathbb{R}^{2}\right) ; \quad U \in L^{2}\left([0, T] \times \Omega_{x}, H^{-1}\left(\mathbb{R}_{v}^{n}\right)\right) ; \\
& E(x, t) \in L^{\infty}\left([0, T], L^{p}\left(\mathbb{R}_{x}^{2}\right)\right),
\end{aligned}
$$

where $p>2$. Then equation (2.1) has a unique solution $f$ in a class of functions $Y$ defined according to

$$
Y=\left\{f \in L^{2}\left([0, T] \times \Omega_{x}, H^{1}\left(\mathbb{R}_{v}^{2}\right)\right), \forall \text { fixed } x \in \Omega, \frac{\partial f}{\partial t}+v \cdot \nabla_{x} \in L^{2}\left([0, T], H^{-1}\left(\mathbb{R}_{v}^{2}\right)\right)\right\} .
$$

Proof Since $E(x, t) \in L_{t}^{\infty} L_{x}^{p}, \iint \nabla_{\nu} \varphi \cdot E \varphi d x d v$ exists for any $\varphi \in \mathcal{D}$, which implies $\int \nabla_{\nu} \varphi$. $E \varphi d v=0$.

On the other hand, since $f \in L^{2}\left([0, T] \times \Omega_{x}, H^{1}\left(\mathbb{R}_{v}^{2}\right)\right)$, we have $\forall x \in \Omega, \nabla_{v} f \in L^{2}([0, T]$, $H^{-1}\left(\mathbb{R}_{v}^{2}\right)$ ), which implies $\forall x \in \Omega$,

$$
\frac{\partial f}{\partial t}+v \cdot \nabla_{x} f=U-E \cdot \nabla_{v} f+\sigma \Delta_{v} f \in L^{2}\left([0, T], H^{-1}\left(\mathbb{R}_{v}^{2}\right)\right) .
$$

The other processes are similar.

Remark 2.2 Similar to Lemma A.1 on p.535 of [5], we mean the initial condition that for any $u \in Y, u$ admits trace value $u(x, v, 0) \in L_{v}^{2}$ for a.e. $x \in \Omega$.

Lemma 2.3 Assume in addition $f_{0} \in L_{x}^{p} L_{v}^{1} ; U \in L_{t}^{1} L_{x}^{p} L_{v}^{1}$, then the solution defined in Lemma 2.1 belongs to $L^{\infty}\left([0, T], L_{x}^{p} L_{v}^{1}\right)$ and satisfies for $t \in[0, T]$ a.e.,

$$
\|f(t)\|_{L_{x}^{p} L_{v}^{1}} \leq\left\|f_{0}\right\|_{L_{x}^{p} L_{v}^{1}}+\int_{0}^{t}\|U(s)\|_{L_{x}^{p} L_{v}^{1}} d s .
$$

Proof Using quite a similar method to that on p.540 of [5], we get for a.e. $x$,

$$
\|f(t, x)\|_{L_{v}^{1}} \leq\left\|f_{0}\right\|_{L_{\nu}^{1}}+\int_{0}^{t}\|U(s)\|_{L_{v}^{1}} d s .
$$

Taking the $L^{p}$ norm with respect to $x$ yields the desired result. 


\section{Proof of the main theorem}

After the above preparation, we are in a position to prove our main theorem.

Proof To prove the theorem, we split the process into several steps. The first step is to construct the approximating solution sequence; in the second step, we prove the regularity of the solution we have obtained; and in the last step, we prove the uniqueness. To get the existence of a weak solution in the space $L_{x}^{p}$, it is natural to require $f^{n}$ to be a Cauchy sequence in the strong topology of $L_{x}^{p}$ and $E(t, x)$ to be bounded in $L_{x}^{p^{\prime}}$, here $\frac{1}{p}+\frac{1}{p^{\prime}}=1$. To estimate the $L^{p^{\prime}}$ norm of $E(t, x)$, we will use the Hardy inequality.

Step 1: We construct an iterative solution sequence to approximate the solution of the original equation.

$$
\left\{\begin{array}{l}
\frac{\partial f^{n+1}}{\partial t}+v \cdot \nabla_{x} f^{n+1}+\left.E^{n}\right|_{\Omega} \cdot \nabla_{v} f^{n+1}-\sigma \Delta_{v} f^{n+1}=0 \\
E^{n}(x, t)=\int_{\mathbb{R}^{2}} \frac{x-y}{|x-y|^{2}} \rho^{n}(y, t) d y \\
\rho^{n}(x, t)=\int_{\mathbb{R}^{2}} f^{n}(x, v, t) d v \\
f^{n+1}(x, v, 0)=f_{0}(x, v) \\
\left.\left\|f^{n+1}\right\|_{L_{\nu}^{1}}\right|_{\partial \Omega}=0
\end{array}\right.
$$

Firstly, on the one hand, to guarantee the weak convergence, we need the weak convergence of $E^{n}$ in $L^{p^{\prime}}$, which in turn can be deduced by the boundedness of $E^{n}$ in $L^{p^{\prime}}$. By the Hardy inequality [10-12], we have

$$
\left\|E^{n}\right\|_{L^{p^{\prime}}} \leq\left\|\frac{1}{|x|} * \rho_{x}^{n}\right\|_{L^{p^{\prime}}} \leq C\left\|f^{n}\right\|_{L_{x}^{p} L_{v}^{1}},
$$

where

$$
\frac{1}{p}+\frac{1}{2}=1+\frac{1}{p^{\prime}}
$$

On the other hand, by Lemma 2.1 and Lemma 2.3, let $f^{0}=0$, we obtain a unique solution $f^{n+1}$ to (3.1). To pass to the limit in the system of (1.1), we need a strong convergence. In order to get this, we compute the Cauchy sequence as follows:

$$
\begin{aligned}
& \left\|f^{n+1}(x, v, t)-f^{n}(x, v, t)\right\|_{L_{x}^{p} L_{v}^{1}} \\
& \leq \int_{0}^{t}\left\|\left(E^{n}(x, s)-E^{n-1}(x, s)\right)\right\| \nabla_{v} f^{n}\left\|_{L_{v}^{1}}\right\|_{L_{x}^{p}} d s \\
& \leq \int_{0}^{t}\left\|\frac{1}{|x|} *\left|\rho^{n}-\rho^{n-1}\right|\right\| \nabla_{v} f^{n}\left\|_{L_{v}^{1}}\right\|_{L_{x}^{p}} d s \\
& \leq \int_{0}^{t} \int_{\mathbb{R}_{y}^{2}}\left|\rho^{n}(y, s)-\rho^{n-1}(y, s)\right| d y \\
& \quad \cdot\left(\int_{\mathbb{R}_{x}^{2}}\left(\frac{1}{|x-y|^{n-1}}\left\|\nabla_{v} f^{n}(x, v, s)\right\|_{L_{v}^{1}}\right)^{p} d x\right)^{\frac{1}{p}} d s \\
& \leq \int_{0}^{t}\left\|\rho^{n}(\cdot, s)-\rho^{n-1}(\cdot, s)\right\|_{L_{x}^{p}}\left\|\left(\frac{1}{|x|^{p}} * x_{x}\left\|\nabla_{v} f^{n}(x, v, s)\right\|_{L_{v}^{1}}^{p}\right)^{\frac{1}{p}}\right\|_{L_{x}^{p^{\prime}}} d s .
\end{aligned}
$$


Here

$$
\frac{1}{p}+\frac{1}{p^{\prime}}=1
$$

Combining (3.2) with (3.3), we solve the indices $p=\frac{4}{3}$ and $p^{\prime}=4$. We claim that

$$
\left\|\left(\frac{1}{|x|^{p}} \begin{array}{c}
* \\
x
\end{array}\left\|\nabla_{v} f^{n}(x, v, s)\right\|_{L_{v}^{1}}^{p}\right)^{\frac{1}{p}}\right\|_{L_{x}^{p^{\prime}}} \leq C
$$

uniformly in $n$. Thus,

$$
\left\|f^{n+1}(x, v, t)-f^{n}(x, v, t)\right\|_{L_{x}^{p} L_{v}^{1}} \leq \int_{0}^{t}\left\|f^{n}(\cdot, s)-f^{n-1}(\cdot, s)\right\|_{L_{x}^{p} L_{v}^{1}}
$$

By a process of induction, we obtain

$$
\left\|\left(f^{n+1}-f^{n}\right)(x, v, t)\right\|_{L_{x}^{p} L_{v}^{1}} \leq \frac{C^{n} t^{n}}{n !} \max _{t \in[0, T]}\left\|\left(f^{1}-f^{0}\right)(x, v, t)\right\|_{L_{x}^{p} L_{v}^{1}},
$$

which implies $f^{n}$ converges to some $f$ in $L_{\text {loc }}^{\infty}\left([0, T), L_{x}^{p} L_{v}^{1}\right)$.

Proof of the claim of (3.4)

A direct calculation by using the Hardy inequality yields

$$
\begin{aligned}
& \left\|\left(\frac{1}{|x|^{p}} \underset{x}{*}\left\|\nabla_{v} f^{n}(x, v, s)\right\|_{L_{v}^{1}}^{p}\right)^{\frac{1}{p}}\right\|_{L_{x}^{p^{\prime}}} \\
& \quad \leq C\|\| \nabla_{v} f^{n}(x, v, s)\left\|_{L_{v}^{1}}^{p}\right\|_{L_{x}^{q}} \\
& \quad=C\left\|\nabla_{v} f^{n}(x, v, s)\right\|_{L_{x}^{p q} L_{v}^{1}},
\end{aligned}
$$

here $\frac{1}{q}+\frac{p}{2}=1+\frac{p}{p^{\prime}}, q>1$

Differentiating equation (3.1) by $D_{x}$ and $D_{v}$, respectively, we get

$$
\left\{\begin{array}{l}
\frac{\partial\left(D_{x} f^{n+1}\right)}{\partial t}+v \cdot \nabla_{x}\left(D_{x} f^{n+1}\right)+E^{n} \cdot \nabla_{v}\left(D_{x} f^{n+1}\right)-\sigma \Delta_{v}\left(D_{x} f^{n+1}\right)=-\nabla_{x} E^{n} \cdot \nabla_{v} f^{n+1} \\
\frac{\partial\left(D_{v} f^{n+1}\right)}{\partial t}+v \cdot \nabla_{x}\left(D_{v} f^{n+1}\right)+E^{n} \cdot \nabla_{v}\left(D_{v} f^{n+1}\right)-\sigma \Delta_{v}\left(D_{v} f^{n+1}\right)=-\nabla_{x} f^{n+1} .
\end{array}\right.
$$

Applying Lemma 2.3 to (3.6) yields

$$
\left\{\begin{array}{l}
\left\|\nabla_{x} f^{n+1}\right\|_{L_{x}^{p} L_{v}^{1}} \leq\left\|\nabla_{x} f_{0}\right\|_{L_{x}^{p} L_{v}^{1}}+\int_{0}^{t}\left\|\nabla_{x} E^{n} \cdot \nabla_{v} f^{n+1}\right\|_{L_{x}^{p} L_{v}^{1}} d s \\
\left\|\nabla_{v} f^{n+1}\right\|_{L_{x}^{p} L_{v}^{1}} \leq\left\|\nabla_{v} f_{0}\right\|_{L_{x}^{p} L_{v}^{1}}+\int_{0}^{t}\left\|\nabla_{x} f^{n+1}\right\|_{L_{x}^{p} L_{v}^{1}} d s,
\end{array}\right.
$$

where $p>1$. Since

$$
\begin{aligned}
& \left\|\nabla_{x} E^{n} \cdot \nabla_{v} f^{n+1}\right\|_{L_{x}^{p} L_{v}^{1}} \leq\left\|\nabla_{x} E^{n}\right\|_{L_{x}^{p_{1}}}\left\|\nabla_{v} f^{n+1}\right\|_{L_{x}^{p_{2}} L_{v}^{1}}, \quad \frac{1}{p_{1}}+\frac{1}{p_{2}}=\frac{1}{p}, \\
& \left\|\nabla_{x} E^{n}\right\|_{L_{x}^{p_{1}}} \leq\left\|\frac{1}{|x|^{N-1}} * \nabla_{x} \rho^{n}\right\|_{L_{x}^{p_{1}}} \leq C\left\|\nabla_{x} f^{n}\right\|_{L_{x}^{p q} L_{v}^{1}}, \quad \frac{1}{p q}+\frac{1}{2}=1+\frac{1}{p_{1}} .
\end{aligned}
$$


We require $p_{1} \leq p^{\prime}, p<p^{\prime}$, this is an easy thing. Note that $L^{p q}(\Omega) \subset L^{p}(\Omega)$ and $L^{p_{2}}(\Omega) \subset$ $L^{p}(\Omega)$. Plugging (3.8) and (3.9) into (3.7) yields

$$
\left\{\begin{array}{l}
\left\|\nabla_{x} f^{n+1}\right\|_{L_{x}^{p} L_{\nu}^{1}} \leq\left\|\nabla_{x} f_{0}\right\|_{L_{x}^{p} L_{\nu}^{1}}+\int_{0}^{t}\left\|\nabla_{x} f^{n}\right\|_{L_{x}^{p} L_{\nu}\left\|\nabla_{v} f^{n+1}\right\|_{L_{x}^{p} L_{v}^{1}} d s} \\
\left\|\nabla_{v} f^{n+1}\right\|_{L_{x}^{p} L_{v}^{1}} \leq\left\|\nabla_{v} f_{0}\right\|_{L_{x}^{p} L_{v}^{1}}+\int_{0}^{t}\left\|\nabla_{x} f^{n+1}\right\|_{L_{x}^{p} L_{v}^{1}} d s .
\end{array}\right.
$$

Next, we only have to solve the Gronwall inequalities in the form

$$
\left\{\begin{array}{l}
f(t) \leq C_{0}+\int_{0}^{t} f(s) g(s) d s \\
g(t) \leq C_{1}+\int_{0}^{t} f(s) d s
\end{array}\right.
$$

These inequalities only hold in finite time, we denote the maximal existence time by $T$, i.e., for $t \in[0, T)$, there exists $\alpha(t) \in L^{\infty}([0, T))$ such that

$$
\left\|\nabla_{v} f^{n}(t)\right\|_{L_{x}^{p} L_{v}^{1}} \leq \alpha(t), \quad\left\|\nabla_{x} f^{n}(t)\right\|_{L_{x}^{p} L_{v}^{1}} \leq \alpha(t)
$$

Therefore, the claim of (3.4) holds.

According to the standard weak convergence process, we conclude that $f$ is a solution of the Cauchy problem of equations of (1.1).

Step 2: Regularity of the solution.

Denote $D_{x}$ or $D_{v}$ by $D$. Since $f^{n} \rightarrow f$ in $L_{x}^{p} L_{v}^{1}$, which deduces $D f^{n} \rightarrow D f$ in $\mathcal{D}^{\prime}\left(\Omega_{x} \times \mathbb{R}_{v}^{2}\right)$, note that $\left\|D f^{n}\right\|_{L_{x}^{p} L_{v}^{1}} \leq$ const, we have $\|D f\|_{L_{x}^{p} L_{v}^{1}} \leq\left\|D f^{n}\right\|_{L_{x}^{p} L_{v}^{1}} \leq$ const.

By property (i) of Proposition A.3 in [5], we conclude that $f^{n}$ is nonnegative. Moreover, $f^{n} \rightarrow f$, a.e. $(x, v) \in \Omega_{x} \times \mathbb{R}_{v}^{2}$, since $f^{n} \rightarrow f$ in $L_{x}^{p} L_{v}^{1}$, which implies that $f$ is nonnegative.

Step 3: Uniqueness of the solution.

The uniqueness is a direct consequence of

$$
\|(f-\tilde{f})(x, v, t)\|_{L_{x}^{p} L_{v}^{1}} \leq C \int_{0}^{t}\|(f-\tilde{f})(x, v, t)\|_{L_{x}^{p} L_{v}^{1}}
$$

which in turn is a result of a very similar process to (3.5).

Next we are going to deal with the boundary, i.e., to show that the solution satisfied the boundary condition.

On the one hand, by Lemma 2.3, we have $\left\|f^{n}\right\|_{L_{\nu}^{1}} \leq\left\|f_{0}\right\|_{L_{\nu}^{1}}$ for $x$ a.e.; on the other hand, $\left\|f^{n}(t, x, v)\right\|_{L_{v}^{1}} \rightarrow\|f(t, x, v)\|_{L_{v}^{1}}$ for $x$ a.e. Thus $\|f(t, x, v)\|_{L_{v}^{1}} \leq\left\|f_{0}\right\|_{L_{v}^{1}}$ for $x$ a.e., note the assumption of $\left.\left\|f_{0}\right\|_{L_{v}^{1}}\right|_{\partial \Omega}=0$ in the sense of trace of $L_{x}^{p}$, we conclude in the sense of trace of $L_{x}^{p}$, since the boundary is $C^{1}$ and $\left\|f^{n}\right\|_{L_{v}^{1}} \in W_{x}^{1, p}$ :

$$
\left.\|f(t, x, v)\|_{L_{v}^{1}}\right|_{\partial \Omega}=0
$$

The authors declare that they have no competing interests.

\section{Authors' contributions}

JCC is in charge of Section 2 and participated in the computation and discussion of the main theorem, Section 3. CH is responsible for the Section 3. This paper was finished under our joint efforts. We made many modifications and approve the final manuscript together. All authors read and approved the final manuscript. 


\section{Author details}

'School of Mathematics and Statistics, Central China Normal University, Wuhan, 430079, P.R. China. ${ }^{2}$ School of Mathematics and Statistics, Wuhan University, Wuhan, 430072, P.R. China.

\section{Acknowledgements}

The authors would like to thank the editors and the reviewers for providing us with valuable and detailed comments and suggestions which have brought great improvements to this manuscript. Also, they would like to thank Prof. C. J. Zhu (CCNU, School of Mathematics and Statistics) and Dr. H. Y. Wen (CCNU, School of Mathematics and Statistics) for helpful discussions and suggestions very much.

\section{Received: 26 June 2013 Accepted: 14 October 2013 Published: 08 Nov 2013}

\section{References}

1. Yang, T, Yu, HJ: Global classical solutions for the Vlasov-Maxwell-Fokker-Planck system. SIAM J. Math. Anal. 42(1), 459-488 (2010)

2. Triolo, L: Global existence for the Vlasov-Poisson-Fokker-Planck equation in many dimensions, for small data. Math. Methods Appl. Sci. 10, 487-497 (1988)

3. Neunzert, H, Pulvirenti, M, Triolo, L: On the Vlasov-Fokker-Planck equation. Math. Methods Appl. Sci. 6, $527-538$ (1984)

4. Zheng, Y, Majda, A: Existence of global weak solutions to one-component Vlasov-Poisson and Fokker-Planck-Poisson systems in one space dimension with measures as initial data. Commun. Pure Appl. Math. 47(10), 1365-1401 (1994)

5. Degond, P: Global existence of smooth solutions for the Vlasov-Fokker-Planck equation in 1 and 2 space dimensions. Ann. Sci. Éc. Norm. Super., 19(4), 519-542 (1986)

6. Yan, G: Decay and continuity of the Boltzmann equation in bounded domains. Arch. Ration. Mech. Anal. 197, 713-809 (2010)

7. Hwang, HJ: Regularity for the Vlasov-Poisson system in a convex domain. SIAM J. Math. Anal. 36(1), 121-171 (2004)

8. Strain, RM: The Boltzmann equation, Besov spaces, and optimal time decay rates in $\mathbb{R}_{x}^{n}$. arXiv:1206.0027v1

9. Evans, LC: Partial Differential Equations. Graduate Studies in Mathematics. Am. Math. Soc., Providence (1998)

10. Radulescu, V, Smets, D, Willem, M: Hardy-Sobolev inequalities with remainder terms. Topol. Methods Nonlinear Anal. 20(1), 145-149 (2002)

11. Brezis, H, Marcus, M: Hardy's inequalities revisited. Ann. Sc. Norm. Super. Pisa, Cl. Sci. (4) 25(1-2), $217-237$ (1997)

12. Bahouri, H, Chemin, J-Y, Danchin, R: Fourier Analysis and Nonlinear Partial Differential Equations. Springer, Berlin (2011)

10.1186/1687-2770-2013-233

Cite this article as: Chen and He: On local existence of the Vlasov-Fokker-Planck equation in a 2D anisotropic space. Boundary Value Problems 2013, 2013:233

\section{Submit your manuscript to a SpringerOpen ${ }^{\circ}$ journal and benefit from:}

- Convenient online submission

- Rigorous peer review

- Immediate publication on acceptance

- Open access: articles freely available online

- High visibility within the field

- Retaining the copyright to your article 